\title{
Examination of polarization coupling in a plucked musical instrument string via experiments and simulations
}

\author{
Alexander Brauchler, Pascal Ziegler, and Peter Eberhard* \\ Institute of Engineering and Computational Mechanics, University Stuttgart, Pfaffenwaldring 9, 70569 Stuttgart, Germany
}

Received 2 April 2020, Accepted 4 June 2020

\begin{abstract}
In this article, the transient motion of a realistically plucked guitar string is studied experimentally and numerically in both transversal polarizations. The frequency dependent damping and suitable initial conditions are identified in the experiment and used in a simulation. For this reason an experimental set-up consisting of a string, an excitation mechanism and two laser Doppler vibrometers is developed. The excitation mechanism performs a realistic and reproducible plucking motion with a plectrum. Two laser Doppler vibrometers are used to measure the string oscillation transversally in two polarizations. The experimental set-up makes it possible to measure the string's motion under reproducible conditions and, hence, at different positions for the same oscillation. This capability renders the identification of suitable initial conditions, i.e., initial displacement and velocity as well as the pre-tension, for a string model possible. Furthermore, a finite element model for the string is developed that takes into account the oscillation in both transversal planes of polarization and the coupling between them. Finally, the model results are in good agreement with the measurements. With help of the numerical model it can be vividly shown that the coupling between the polarizations of the oscillation is due to a torsional movement of the string on the saddle.
\end{abstract}

Keywords: Guitar string vibration, Musical instruments, Realistic plucking, LDV measurement, Experimental study, Numerical study

\section{Introduction}

The acoustic guitar is a popular string instrument in which the sound results from a mechanical process beginning with the oscillation of the plucked string. The sound of the string is influenced by several physical effects like dispersion and damping which are difficult to describe and consider. Dispersion results from the bending stiffness of the string and shifts the overtones to relatively higher frequencies. Moreover, the frequency dependent damping of the string influences the oscillation to a large extend [1].

The basic mathematical model for a vibrating string is the one-dimensional wave equation which can be found in many textbooks. Usually, this is extended by additional terms to include physical effects like damping or dispersion [2-4]. A comparison of different stiff string models can be found in Ducceschi and Bilbao [5]. Besides finite difference simulations of these stiff string models $[6,7]$ digital waveguide models are a common approach $[8,9]$.

Specifically for the guitar, the work of Woodhouse is to mention [1, 10]. In these publications three numerical models, namely frequency-domain, time-domain and modal superposition methods, for the acoustic guitar are compared to each other and to experiments. However, the results compared to the experiment were not satisfying for a transient measurement of the string oscillation.

Recent sophisticated string models contain the aforementioned physical features and show very realistic results when compared to experiments. For example one study is engaged in piano modeling and is using a Timoshenko beam model which shows a good result for the string motion when compared to an experiment [11]. The piano strings are struck and not plucked and only one transversal polarization of the string oscillation is excited and included in the model. Furthermore, contacts with the fretboard of a guitar are included in models and the simulation results are shown to be in very good agreement with experiments $[12,13]$. However, to make a comparison with an experimental measurement possible, the string was excited using a copper wire in those studies. This method produces simple initial conditions for a numerical model but they are not realistic.

Moreover, many publications exist on the purely experimental measurement of the string oscillation. The methods to measure the oscillation include the electromagnetic pickup [14], an electrodynamic method [15], or electric field

*Corresponding author: peter.eberhard@itm.uni-stuttgart.de 
sensing methods [16] which are applicable to a steel string only. In other publications optical measurement techniques like opto-switch sensors [17], high-speed cameras [18], or laser Doppler vibrometers (LDVs) [19] are used. On the other hand, publications are not only concerned with the string oscillation but also with the player and the plucking motion which has a great influence on the instruments sound. There have been studies about the plucking action investigating the motion of the plectrum and the string in a harpsichord for different plectrum shapes and plucking strengths $[20,21]$. Another study is concerned about the plucking of a harp [22]. Nevertheless, these studies are essentially experimental and do not include a numerical model for the string oscillation after the excitation.

Previous studies found two eigenfrequencies very close to each other for the string oscillation close to the bridge of an instrument [1]. This effect can be explained by the two polarization planes of the string's oscillation having slightly different effective lengths and, hence, different eigenfrequencies. In Bank and Karjalainen [23] it is stated, that the guitar bridge acts as a mechanical coupling device for the two planes of polarization in the string oscillation and causes this effect.

The aim and novel contribution of this study is, to combine the previous findings with a realistically plucked string in a reproducible experiment and a well suited numerical model to approximate the plucked string motion in both transversal polarizations. Furthermore, the coupling of the two transversal polarizations through a plain half-round saddle is examined. To reach this goal, damping parameters and complex initial conditions for the numerical model must be identified from an experiment for one particular string. Therefore, an experimental set-up with an excitation mechanism that yields a reproducible and realistic plucking motion is developed. The results of the numerical model are compared with the experimental results of the realistically plucked string. The novel contribution is not only the method to identify the initial conditions for the entire string using a most realistic, reproducible excitation and their application to the numerical string model, but also the examination of the polarization coupling in a very vivid way through the numerical model. Finally, this procedure results in a very good agreement of numerical simulation and experimental measurement.

\section{Experimental study}

The sound of an acoustic guitar results from an interaction between the guitar body and the string through the bridge. In this work the string shall be examined without any influence from the guitar body. This is why a single string is mounted on guitar-like saddles without a body. The experiment is carried out firstly to reproducibly measure the oscillation of the string under realistic plucking conditions. Secondly, the frequency dependent damping ratio shall be identified, and thirdly, the coupling of the two polarizations of the string is examined. Furthermore, suitable initial conditions for a simulation are identified.

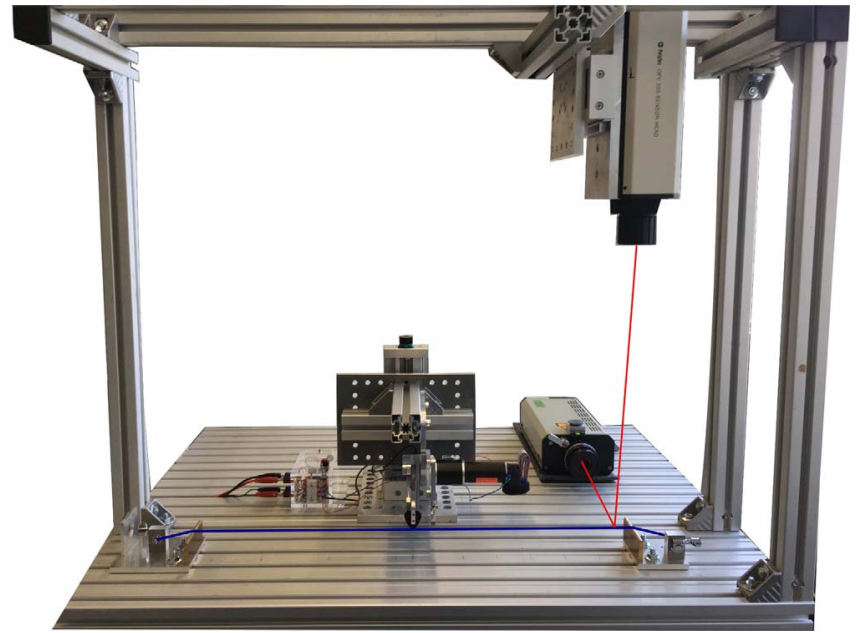

Figure 1. Experimental set-up of the string with two LDVs and automatic excitation mechanism.

\subsection{Experimental set-up}

In the center of the experiment is a bare steel string of an electrical guitar set (Harley Benton Valuestrings 010) with diameter $d=0.43 \mathrm{~mm}$ (third string), which is highlighted in blue in Figure 1. It is clamped between two equal brass saddles assembled in a guitar typical distance of $L=0.65 \mathrm{~m}$. The string can be tuned to the desired tension with a machine head from a guitar. The measurement is carried out with two LDVs of type Polytec OFV 303. One of them measures velocity and displacement of the string at a certain position in horizontal direction while the other one measures the vertical displacement and velocity at the same position. The LDVs allow an accurate measurement of even very small displacements of the string in both transversal polarizations.

Another central part of the experimental set-up is the excitation mechanism displayed in Figure 2. The mechanism was built to replicate a human-like plucking of the string with a plectrum [24]. For the experiments throughout this paper a thin standard plectrum with thickness $d_{\mathrm{pl}}=0.46 \mathrm{~mm}$ is used. The motor driven mechanism consists of five parts transforming the circular motion into a realistic plucking movement. Furthermore, the mechanism yields reproducibility in intensity, point of action and direction of the motion [24]. To realize a fine adjustable plucking position and strength the mechanism is mounted on a horizontally and vertically movable mechanical adjuster. Figure 3 shows the mean value and the uncertainty of ten measured displacement signals of the vertical LDV. It is clearly visible that the excitation mechanism produces well-reproducible signals. Reproducible excitation yields the advantage that the string can be excited multiple times at the same position while the measurement can be carried out at different positions.

\subsection{Identification of damping}

Besides measuring the string oscillation at different positions, the frequency dependent damping ratio shall be 

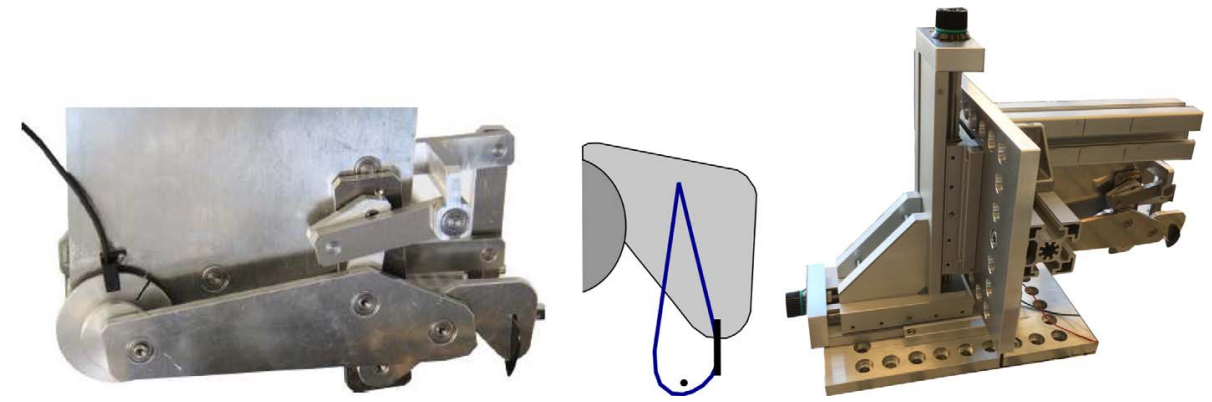

Figure 2. Excitation mechanism (left), schematic path of the plectrum (middle), and application of the mechanism in the experiment (right).

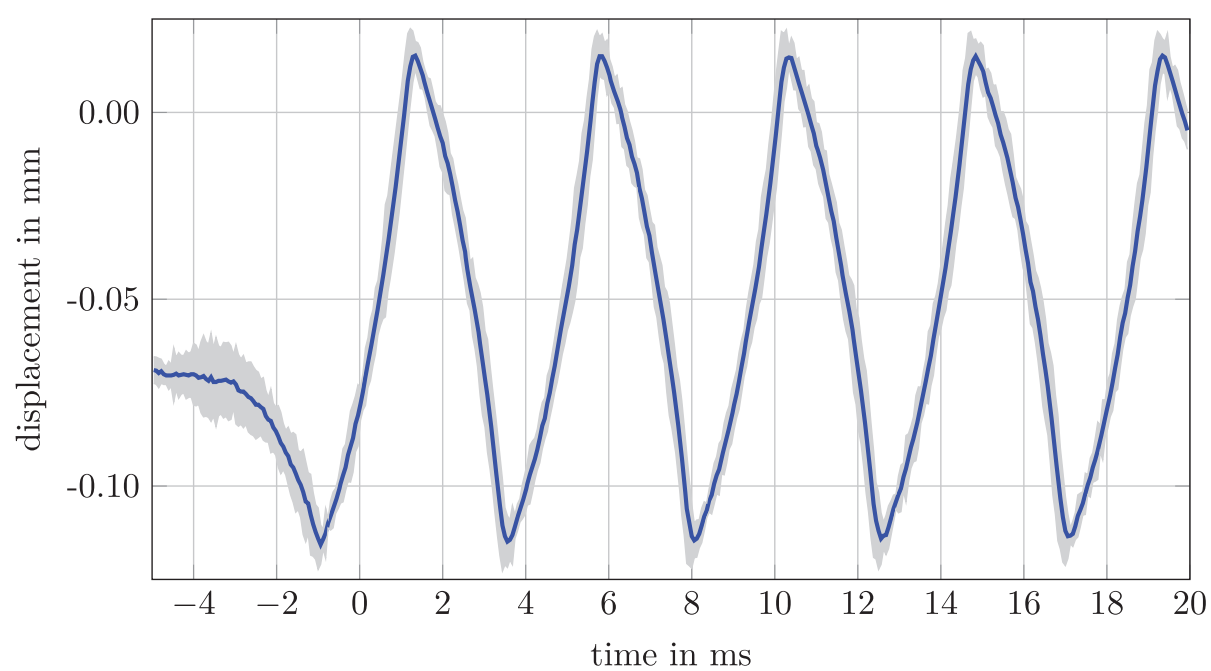

Figure 3. Mean signal and $99.73 \%$ confidence bound from ten measurements. The string is excited in the middle of the string and measured at a position $15 \mathrm{~mm}$ beside the middle.

identified. The damping ratio is identified using the spectrogram. In a spectrogram the short-time Fourier transform of the signal is performed multiple times for successive time frames. The attenuation rates of the string's eigenfrequencies can then be calculated by fitting an exponential decay to the heights of the maxima in these successive time frames. This method was first proposed in Hodges et al. [25] and successfully applied to a guitar string in Mansour [26]. In Figure 4 the resulting damping ratios are displayed up to a frequency of $3 \mathrm{kHz}$. As expected, due to the preferably low coupling to the environment in the experiment, the damping ratios are very low. The minimal damping ratio $\zeta_{\min } \approx 0.01 \%$ is found around $1000 \mathrm{~Hz}$. Earlier studies found the minimal damping ratio at higher frequencies but this seems to be highly dependent on the specific string and the experimental set-up $[12,13,26]$.

With the measured results, a damping model can be fitted for the later use in a simulation. In this paper the common Rayleigh damping model which can be described as:

$$
\zeta=\frac{1}{2}\left(\frac{\alpha}{\omega}+\beta \omega\right)
$$

is used resulting in a frequency dependent damping ratio $\zeta$ with the coefficients $\alpha$ and $\beta$ and frequency $\omega$ (see [27]). The coefficients are then fitted to the experimental data with the least-squares method. Finally this results in the curve presented in Figure 4. The coefficients are found to be $\alpha=0.441 / \mathrm{s}$ and $\beta=2.4 \times 10^{-8} \mathrm{~s}$. In literature, more sophisticated models for the frequency dependent damping of the string exist (see e.g., [28, 29]). However, as can be seen in Figure 4 the damping curve fits fairly well to the measured data in the examined frequency range. The limitations of the Rayleigh damping model are discussed in Section 5.

\subsection{Coupling of polarizations}

When measuring the string's two planes of polarization simultaneously, a small difference in the frequencies of the oscillation is observable. To make the effect visible, a very fine frequency resolution is necessary. In the conducted experiment, the plucked string is measured for $T=10 \mathrm{~s}$ with a sampling rate of $f_{s}=10^{5} \mathrm{~Hz}$. This results in a sufficiently fine frequency resolution of $\Delta f=0.1 \mathrm{~Hz}$. Figure 5 contains the Fourier-transformed signals of the string's 
A. Brauchler et al.: Acta Acustica 2020, 4, 9

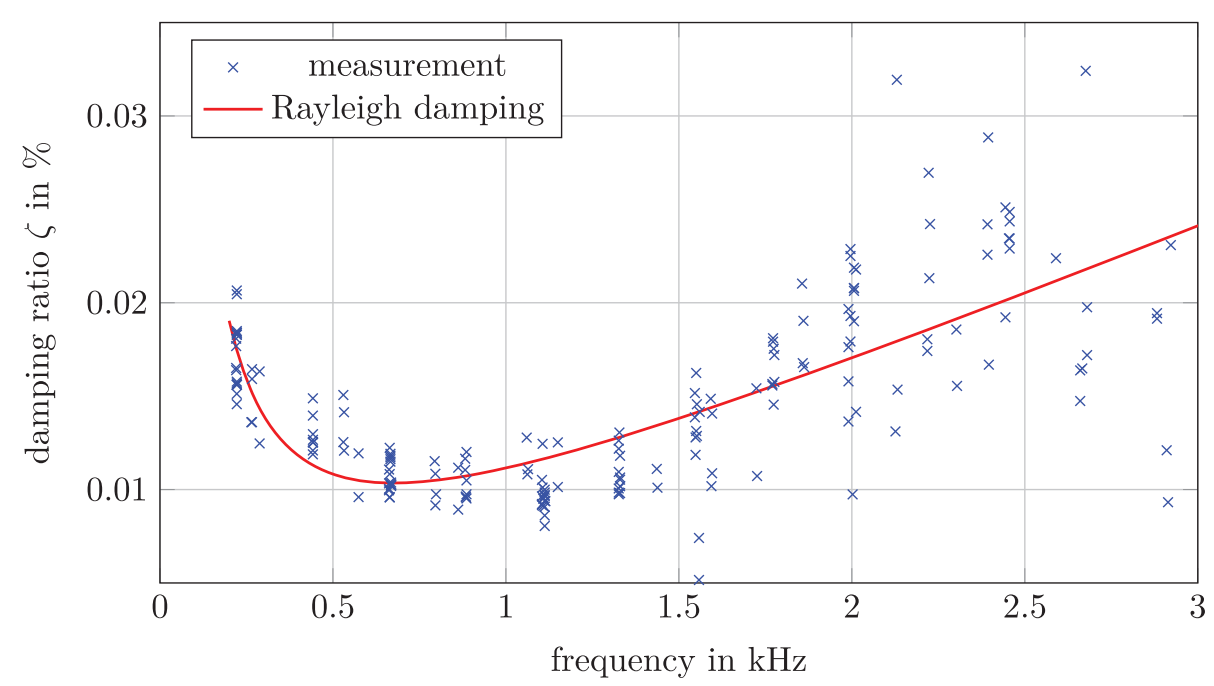

Figure 4. Identified damping ratios over frequency for several experiments and fitted Rayleigh damping curve.
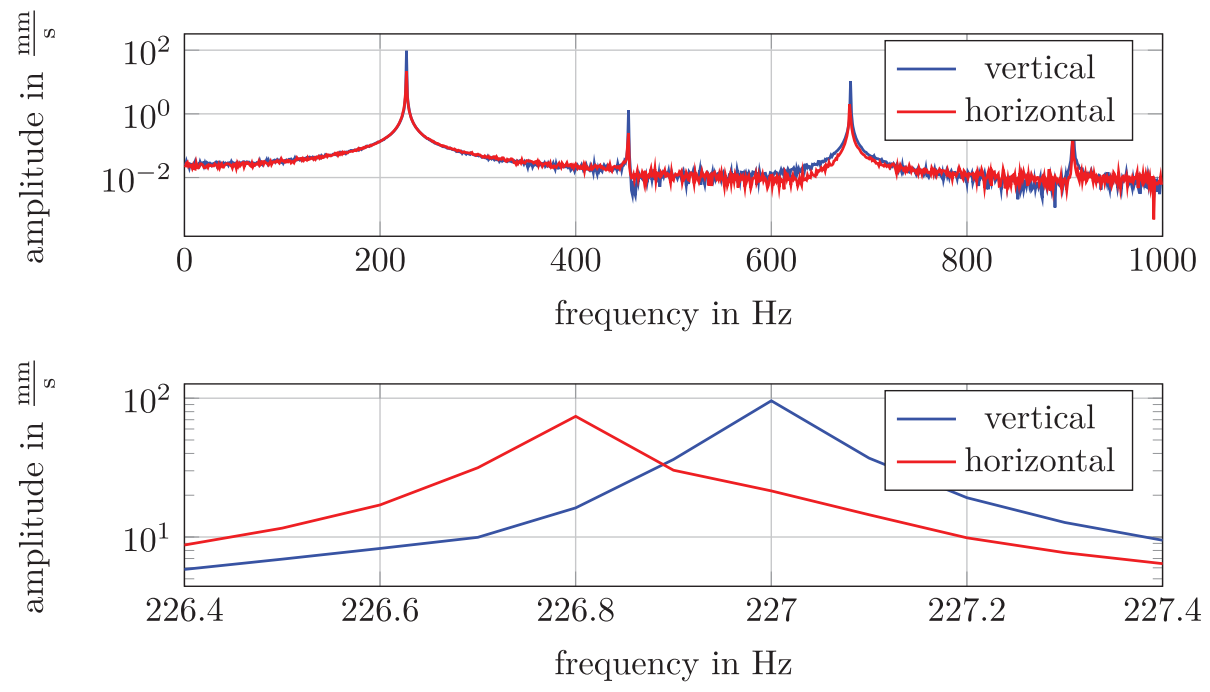

Figure 5. Fourier transformed velocity signal in the two planes of polarization.

velocity in horizontal and vertical polarization. Besides the expected similarity in the upper plot, it is clearly visible in the lower plot that the frequencies of the two planes of polarization deviate from each other by about $0.2 \mathrm{~Hz}$ at the first eigenfrequency.

Another effect is visible when the orbit diagram displaying the vertical oscillation over the horizontal oscillation is examined. Figure 6 shows the transient development of the orbit diagram via four cycles of the oscillation within the first second of free oscillation. Together with a damping effect it is clearly visible that the shape of the orbit varies with time. The shape variation can be explained by dispersion and the small frequency difference between the two planes of polarization.

In literature, multiple possible causes for the frequency difference are found. Firstly, nonlinear behavior due to large amplitudes might couple the polarizations and add further frequencies to the spectrum [26, 30]. Secondly, the saddle might influence the strings oscillation due to coupling of the saddle's vibration with the string's vibration [30]. Furthermore, a stick-slip movement owing to friction or a torsional movement of the string of the string on the saddle might cause the observed effect $[1,26]$.

In all presented measurements the amplitudes of the string oscillation are always smaller than the string's diameter and, therefore, nonlinear effects are very small. Although nonlinear effects like phantom partials cannot be ruled out completely for higher overtones they are very unlikely to cause the observed effect. Moreover, as examined in literature, there is influence of large displacement on the orbit of the string but displacements would have to be larger by at least one order of magnitude to see that 

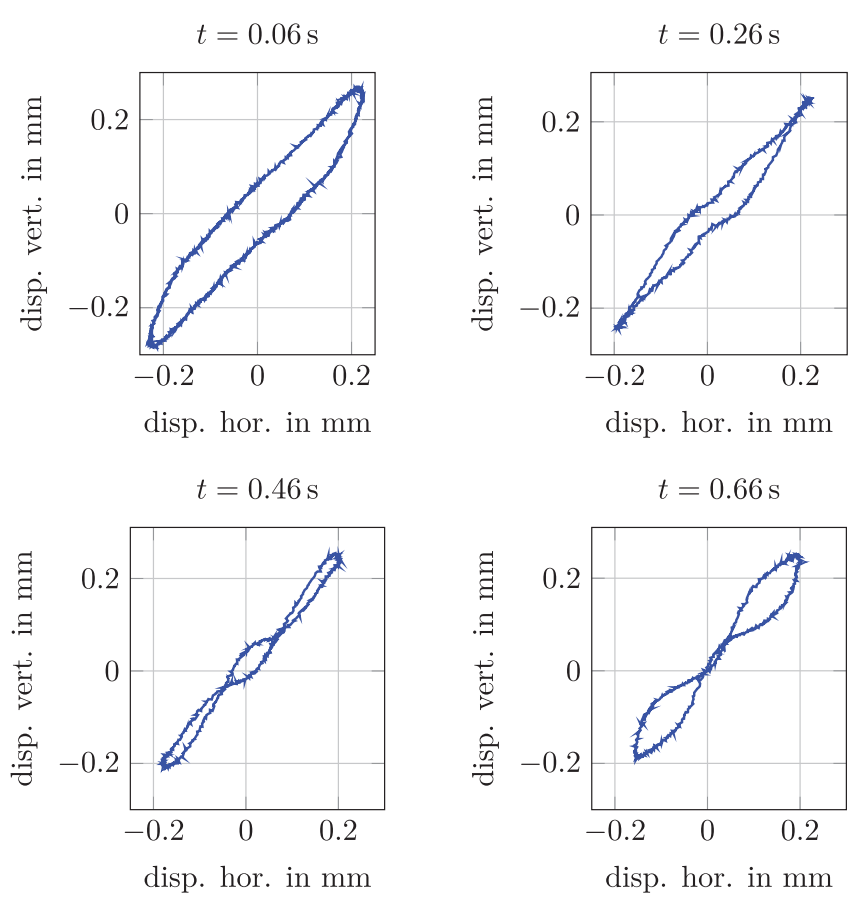

Figure 6. Transient development of the measured orbit diagram at four points in time close to the middle of the string.

influence [30]. Consequently, nonlinear effects are insignificant and can be ruled out as the cause for the observed behavior. If a complete guitar was examined, it would be evident that the oscillation of the bridge influences the oscillation of the string. However, in the presented experiment the saddles are very rigid compared to the string and additional experiments showed clearly that the amplitude of the saddle's vibration is at least two orders of magnitude smaller than the amplitude of the vibration of the string. So in the further numerical investigation, the vibration of the saddles is neglected.

Hence, only stick-slip friction and torsion of the string remain as possible causes for the frequency deviation between the polarizations. Both effects would influence the frequency mainly in horizontal direction due to horizontal movement on the saddle. Yet, preliminary simulations suggested that a string slipping over the saddle causes a different damping behavior than the one observed in the experiments. As the string underwent a slipping movement on the saddle, a high damping in the beginning of the oscillation occured and after a small number of cycles the string would stick on the saddle. Therefore, for a stick-slip movement a decaying frequency difference between the two planes of polarization was observed. On the contrary, the measured string oscillation shows a constant frequency difference and very small damping for a long measurement $(T=10 \mathrm{~s})$ from the beginning until the end. Considering this, the research hypothesis that a torsional movement of the string on the saddle, rather than slipping, causes the frequency difference between the two planes of polarization is concluded. In the following, a numerical model is proposed that is capable of showing vividly that a torsional movement of the string, in fact, causes this behavior while excluding all other possible influences and, hence, this confirms the research hypothesis.

\section{Numerical model}

A realistic string model should include physical effects like damping and dispersion as well as both transversal polarizations of the string oscillation and the coupling between them. Dispersion describes the frequency dependent wave velocity due to bending stiffness of the string leading to slightly higher overtones in reality than the ideal harmonic overtones [2]. The classical wave equation which dates back to D'Alembert does not cover these phenomena. Furthermore, most publications are only modeling a onedimensional string oscillation, but the experimental results described in Section 4 make clear that both transversal polarizations are necessary for a model of a realistically plucked string. Here, a finite element (FE) model of the string is developed using mainly Euler-Bernoulli beam elements in the commercial software Abaqus [31].

\subsection{Mechanical model}

In the following, the FE model is described taking not only physical effects like geometrical stiffness and dispersion into account, but also both planes of polarization and the coupling between them. To include the effect of torsional movement of the string on the saddle, the string model consists of three parts as can be seen in Figure 7. As the string is plucked in the middle, symmetry is used and a half model is created which consists of the main part of the string and a tailpiece discretized with beam elements of type Abaqus B31. In these elements, the Euler-Bernoulli beam model is used which has proven to be a good model for bare steel guitar strings [5, 12]. A Timoshenko beam model would be another option and was investigated by the authors but is avoided here due to numerical issues that might arise and because the Timoshenko model only has physical advantages for thicker strings as for example used in a piano $[5,32]$.

The distribution of eigenfrequencies of a string is dependent on the tension. This stiffening effect due to tension is included in Abaqus when using geometric nonlinearities. Then, also nonlinear effects owing to large displacements are included automatically. As a steel string is used in the experiment, typical material properties for steel are used in the model with Young's modulus $E=210 \mathrm{GPa}$, Poisson ratio $v=0.3$ and density $\rho=7900 \mathrm{~kg} / \mathrm{m}^{3}$. During the dynamic simulation a Hilber-Hughes-Taylor implicit time integrator is used with a maximum time step of $\Delta t=10^{-5} \mathrm{~s}[31]$.

The dimension of the main string and the tailpiece are directly taken from the measured string. This results in a length $l_{\text {main }}=325 \mathrm{~mm}$ of the main part and the length $l_{\text {tail }}=55 \mathrm{~mm}$ for the tailpiece. A round cross section with the string's diameter $d=0.43 \mathrm{~mm}$ is assumed. Both parts of the string are sufficiently fine meshed by 128 elements 


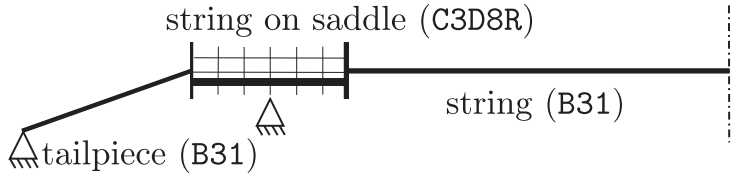

Figure 7. Schematical visualization of the half-string FE model including the coupling between beam and volume elements.

in the half string and 100 elements in the tailpiece. Although the cross section of the string is included in the mathematical model through the moment of inertia, and torsional stress can be calculated, geometrically the diameter of the beam model is zero. Thus, it is not possible to describe a string performing a torsional motion with these beam elements. This is why a small third section describing the part of the string which is in contact with the saddle is modeled and discretized with volume elements of type Abaqus C3D8R. The volume elements yield the advantage that they discretize a three dimensional continuum and, hence, this three dimensional continuum can perform a rotational movement instead of just calculating torsional stress as is done in the beam elements. Of course it would be possible to discretize the whole string with volume elements but just the small section of length $l_{\text {vol }}=1 \mathrm{~mm}$ must already be discretized with 10160 elements. The three parts of the string model are connected via kinematic constraints binding the two lateral degrees of freedom of the adjacent node of the beam element section to the area of the volume element section. Figure 7 shows a schematical visualization of the model with an exaggerated volume part. The boundary conditions at the end of the tailpiece, the right end (middle) of the string and the artificial contact boundary condition are displayed as well in the figure.

On that contact piece discretized with volume elements a small area is defined as contact area with the saddle consisting of only six nodes. In this small area the two translational degrees of freedom orthogonal to the string axis are constrained. For a small rotation, this boundary condition is similar to the string sticking on the saddle. At the end of the tailpiece, which is bended in the same angle of $\varphi=15^{\circ}$ as in the experiment, all degrees of freedom are constrained. It is needless to say that this model comes across quite artificially but with this model it is certainly possible to isolate the effect of a torsional movement of the string on the saddle which might cause the frequency difference and, hence, the specific development of the orbit plot discussed in Section 2.3.

\subsection{Simulation procedure}

The goal of the simulation is to reproduce the string oscillation after the excitation. Therefore, suited initial conditions consisting of an initial displacement and an initial velocity as well as a pre-tension have to be applied to the model [22]. In Abaqus it is not possible to apply the initial conditions and the pre-tension directly in the same computation step. This is why three pre-simulation steps are carried out.
To begin with, the tension is not applied directly but via a longitudinal force on the model that results in a tension. This force can be well approximated by:

$$
F_{L}=\rho \pi\left(L D f_{0}\right)^{2},
$$

where the length $L$, the diameter $D$, the density $\rho$ and the fundamental frequency $f_{0}$ are easy to measure from the experiment [14]. In the simulation the fixed longitudinal boundary condition is removed at the end of the tailpiece and the longitudinal force is applied there. The force is applied with the angle $\varphi=15^{\circ}$ to reach the desired tailpiece angle matching the one in the experiment. A static simulation step is carried out to find the new equilibrium. When the step is finished, the displacements are stored and all degrees of freedom at the supported node are restrained such that the string cannot move longitudinally anymore.

After the first simulation step is carried out, the initial displacement cannot be directly imposed on the nodes of the model anymore. This is why a transversal force is applied on the model in a second static simulation step at the excited node such that the required displacement of the string is obtained. In this case, direction and magnitude of the force are approximated from the results of the experiment. Furthermore, the initial velocity field at the time instant when the free oscillation of the string begins, is obtained. This velocity field identified in Section 4 is applied directly to each node in a subsequent dynamic timestep. After finishing these three pre-simulation steps the actual free oscillation of the string can be simulated.

\section{Experimental identification of initial conditions}

Next, the plucking procedure is examined to identify suitable initial conditions. The main issue here is, finding the time instance where the contact between plectrum and string ends and the free oscillation begins. As a starting point the orbit diagram at a position very close to the excited position displayed in Figure 8 is regarded. In this study the string is plucked in the middle. Measuring exactly at the plucking position is technically not feasible because the excitation mechanism does not leave room for the laser rays. However, the string's behavior at a position very close to the plucking position is assumed to be very similar to that exactly at the plucking point.

The movement of the string during the plucking procedure can be divided into four phases as highlighted in Figure 8. In the beginning, the string sticks to the plectrum (phase 1). After the sticking phase the string and the plectrum show alternately sticking and slipping behavior (phase 2 ). In the third phase the string detaches from the plectrum and finally starts the free oscillation in the fourth phase. In the beginning the string undergoes a slow, nearly horizontal movement indicating that the string is moving with the plectrum. Here the plectrum is only slightly bent. After this phase in which the string sticks to the plectrum, sticking and slipping are alternating as the plectrum is further 


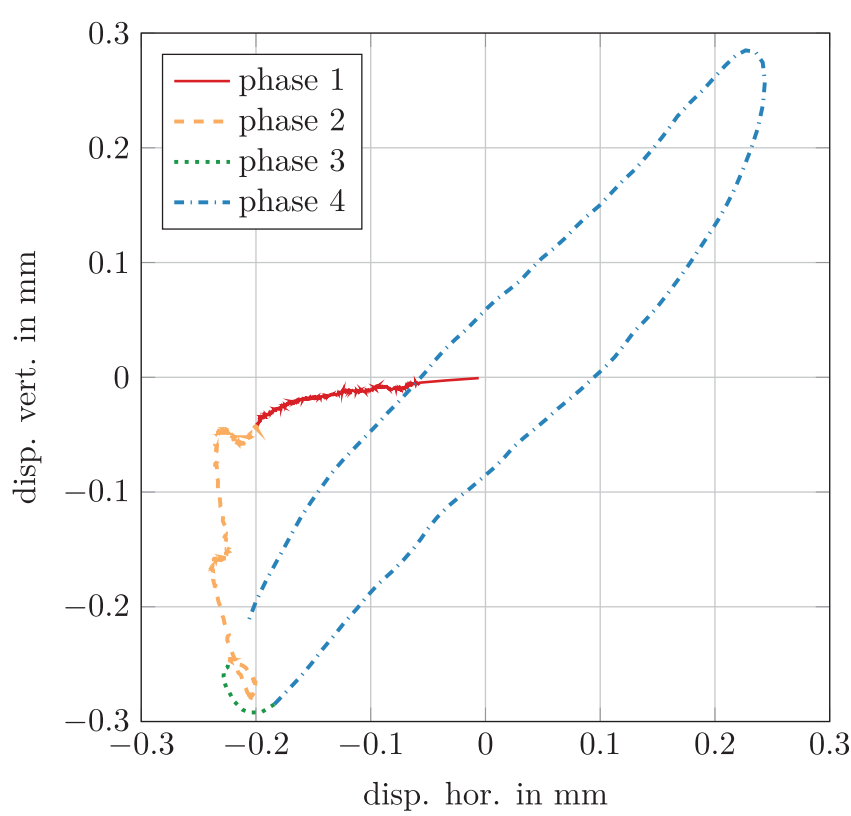

Figure 8. Vertical displacement over horizontal displacement $15 \mathrm{~mm}$ from the plucked position.

bent in the second phase. The slipping is visible in the orbit plot indicated by abrupt movements downwards and slightly in horizontal direction against the plectrum movement. When the string has reached the tip of the plectrum the detachment of the string and the plectrum begins. In the orbit plot it is hard to identify a single specific point in time where the string detaches from the plectrum. This is mainly due to the lack of time information. Nevertheless, identifying a certain time instant is necessary owing to the need for a well defined initial condition for a simulation.

A better insight in the detachment is possible with a plot of the displacement magnitude and velocity magnitude at the same position as visualized in Figure 9. Because the information about the direction is not included in these plots, the stick-slip behavior of the string is hardly visible. It is solely visible in the absolute velocity plot, in which the slipping is indicated by small peaks around the time instances $t=\{-12,-5,-3\} \mathrm{ms}$. On the other hand, the detachment can be found more easily. In the ideal case, one would expect a discontinuous behavior at the detachment point. To be specific, a sharp bend in the absolute displacement plot and a corresponding jump in the absolute velocity are expected. In the absolute displacement plot one can find such a sharp bend shortly after the global maximum at $t \approx-0.1 \mathrm{~ms}$. At the corresponding point in time the gradient in the absolute velocity plot is very high. Accordingly, the detachment instant is defined as the time instant for which the maximum absolute displacement is reached.

In general, initial conditions consisting of initial displacement and initial velocity are necessary for the numerical time integration of the differential equations in the simulation [22]. The initial displacement can be obtained by applying a transversal force at the plucking position statically in a pre-simulation step. This force is then immediately turned off in the instant of detachment [14]. In the following the procedure to identify the initial condition for the velocity from the experimental data is explained. Namely, the goal is to identify a velocity field not only for one specific point but for the whole string. Therefore, in the experiment the string is excited at the same position multiple times while the measured position varies. This is possible due to the reproducibility that the excitation mechanism guarantees. Subsequently, the velocity is interpolated between the measurements and extrapolated for the not measurable part in the middle to obtain a velocity field for the half string model.

In this particular case the string is excited in the middle and the symmetry of the experimental set-up is exploited. Hence, the string is measured at seven positions between the plucked position and one saddle of the set-up. The other half of the string can then be extrapolated. This results in one velocity field for the horizontal direction and one for the vertical direction as well as corresponding displacement fields which are displayed in Figure 10. The identified velocity field in horizontal direction is around zero near the ends of the string and has a clear peak in the middle. A striking fact is the change of sign in the velocity between the axial positions $x=150 \mathrm{~mm}$ and $x=200 \mathrm{~mm}$. This is due to the fact that at this certain time instant the string has just detached from the plectrum in the middle. This causes the middle of the string to move in the opposite direction as the plectrum while the outer part of the string still moves in the same direction as the plectrum. On the contrary, the vertical velocity field shows only small differences between the maximum velocity in the middle of the string and the measured point at the axial position $x=100 \mathrm{~mm}$. Solely at the two most outer measured points the velocity is considerably lower. It can be concluded that the string detaches with a phase shift between the horizontal and vertical movement. This phase shift then leads to the elliptic movement visible in the orbit diagram in Figure 8.

The displacement shape in both polarizations is similar but not identical to the triangular shape that is expected by an ideal pluck [2]. Hence, the approach to apply a single force at the excited position of the string in a static simulation step is well suited to obtain the identified initial displacement. The targeted force is fitted via several static simulations in a trial and error process to match the measured displacement of the string. A good result is achieved with the force:

$$
\mathbf{F}_{T}=\left[\begin{array}{c}
F_{T_{h}} \\
F_{T_{v}}
\end{array}\right]=\left[\begin{array}{l}
-0.104 \\
-0.130
\end{array}\right] \mathrm{N},
$$

consisting of a horizontal transversal part $F_{T_{h}}$ and a vertical transversal part $F_{T_{v}}$. This force is applied statically in a pre-simulation step and turned off before the simulation of the free oscillation begins. Last but not least, the longitudinal force, that results in a tension, is calculated with equation (2). In this specific case the fundamental frequency $f_{0}=189 \mathrm{~Hz}$ has been measured and, thus, the longitudinal force adds up to $F_{L}=70 \mathrm{~N}$. 
A. Brauchler et al.: Acta Acustica 2020, 4, 9
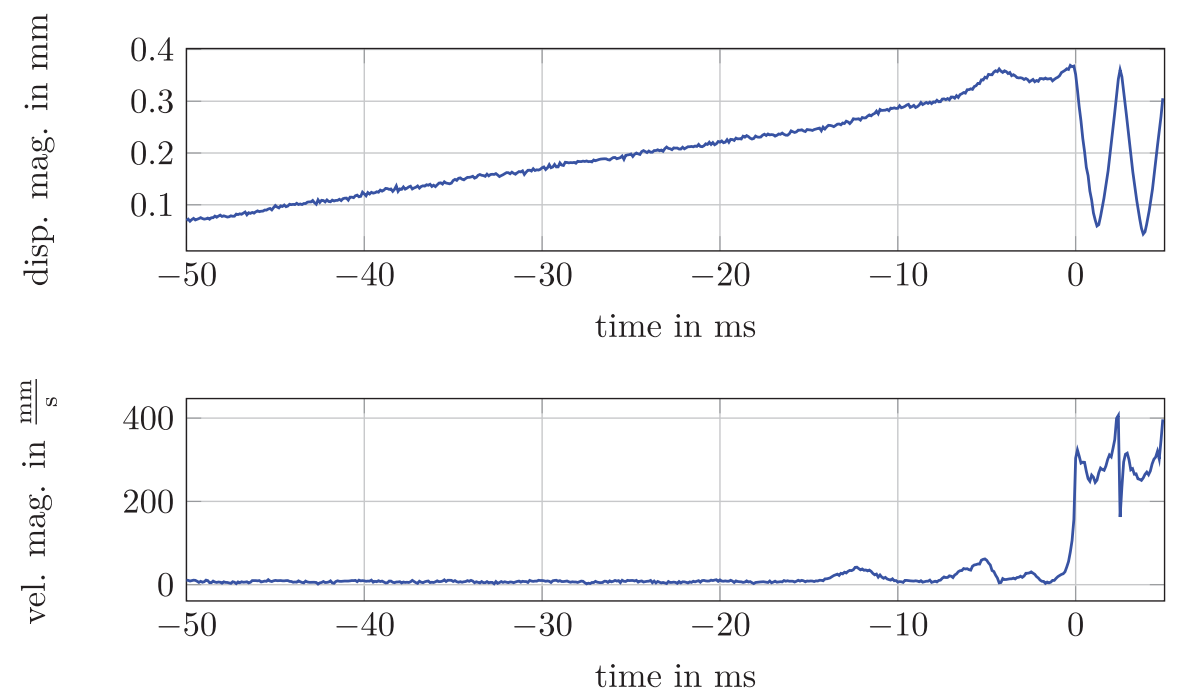

Figure 9. Magnitude of displacement (top) and magnitude of velocity (bottom) of the string measured close to the plucking position.
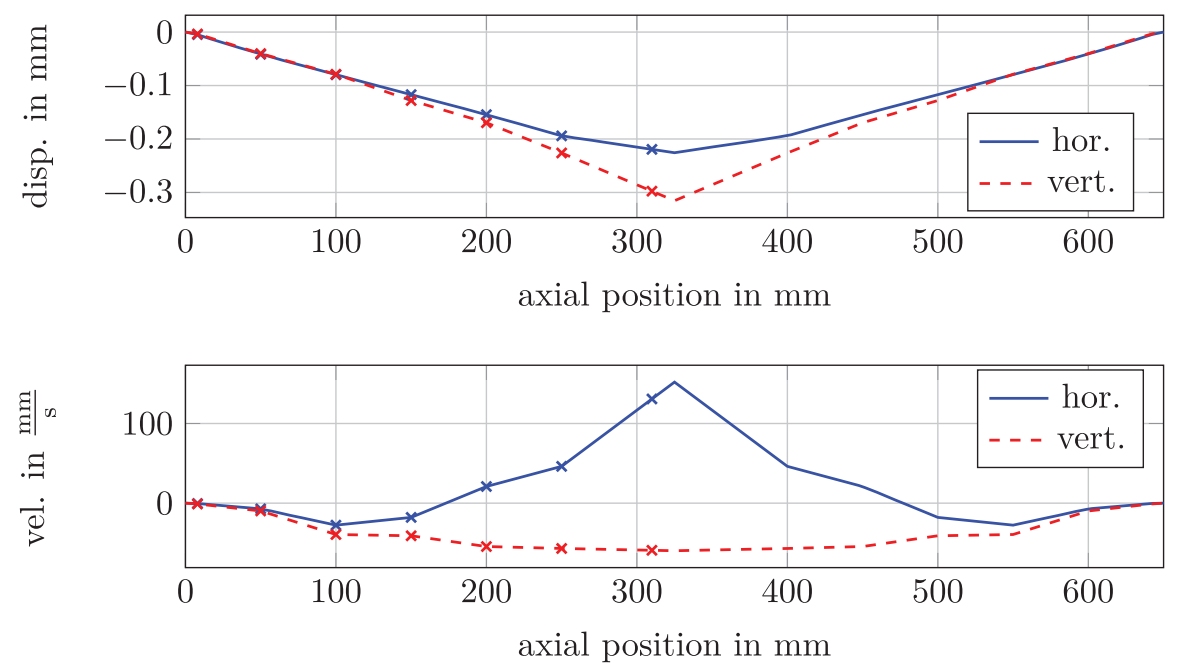

Figure 10. Extrapolated velocity field of the string in horizontal and vertical direction, the measured points are marked.

\section{Comparison of simulation and experiment}

In this section simulation results are shown and compared to the measurements. To begin with, Figure 11 displays the spectrograms of the measured signal and the simulated one at the measured point close to the middle. The eigenfrequencies of the simulation agree with the measured ones. Additionally, both spectrograms have in common that the highest amplitudes correspond to the low odd eigenfrequencies. As the string is plucked in the middle, one would expect the even overtones to vanish completely. While this happens in the simulation, the experimental result contains even overtones although with very low amplitudes. It is worth to note that in some cases, e.g. at the sixth overtone, the amplitude of the even overtones is ascending during the first half of a second. The even overtones existing in the experiment might be caused by small nonlinear effects and because the plectrum might not hit exactly the middle of the string. Furthermore, the identification of the damping ratios for the simulation seems to be successful although limitations of the Rayleigh damping model are visible. While the first odd eigenfrequencies fit well, the eigenfrequency at $1.7 \mathrm{kHz}$ in the measurement is decayed under the threshold of $-90 \mathrm{~dB} / \mathrm{Hz}$ after $0.7 \mathrm{~s}$ while it is still visible after $0.8 \mathrm{~s}$ in the simulation. On the other hand the eigenfrequencies above $2 \mathrm{kHz}$ need roughly twice the time in the experiment that they need in the simulation to decay under the threshold. 

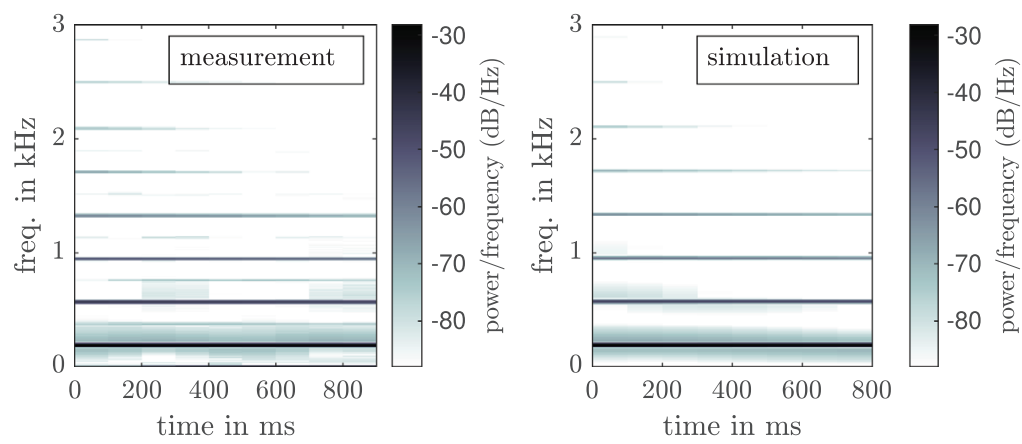

Figure 11. Spectrogram of the measured signal (left) and of the simulated signal (right).
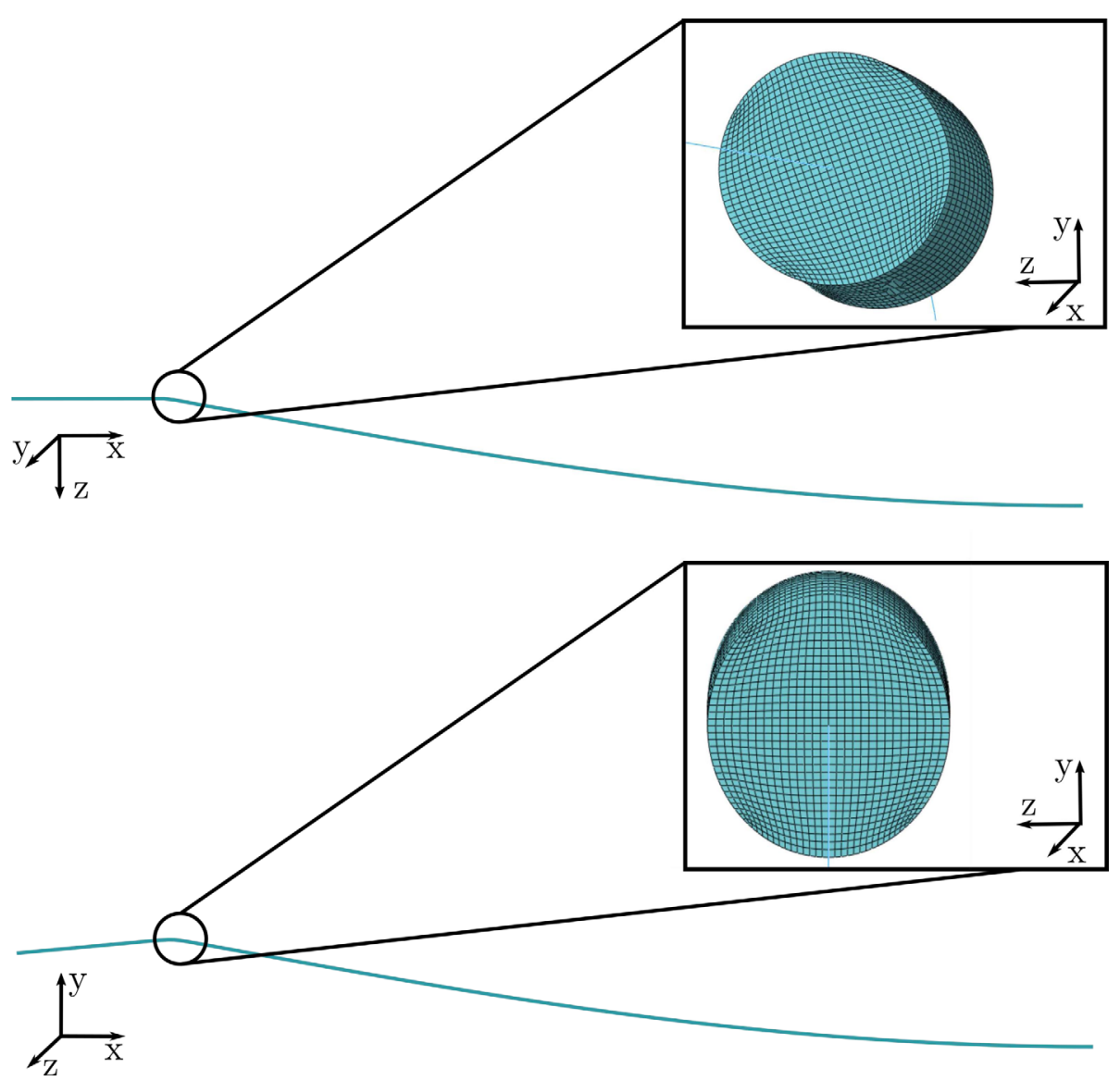

Figure 12. First eigenmodes of the numerical half-string model in horizontal plane (top) and vertical (bottom). The part on the saddle is magnified for both modes.

Secondly, the capability of the numerical model to approximate the torsional movement of the string on the saddle is examined. It is expected, that the torsional movement of the string on the saddle is causing a transient variation of the string's orbit. Figure 12 shows the first two eigenmodes of the numerical string model. In the figure the volume part of the string which is artificially bound to the saddle is displayed as a close up. The first mode is the horizontal first string eigenmode and the second mode is the first string eigenmode in vertical direction.

It is visible by the orientation of the mesh, that a torsion occurs for the horizontal mode. However, the torsion is displayed exaggeratedly. For the identified initial conditions the string is rotated around the longitudinal axis by $\alpha=0.27^{\circ}$ at the position where the volume part of the string is artificially bound to the saddle. For an ideal string it 


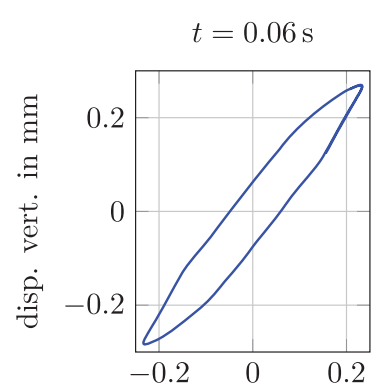

disp. hor. in $\mathrm{mm}$

$$
t=0.26 \mathrm{~s}
$$

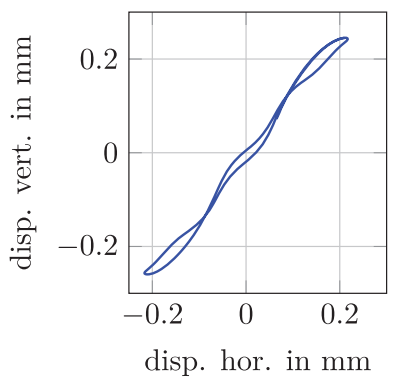

$t=0.16 \mathrm{~s}$

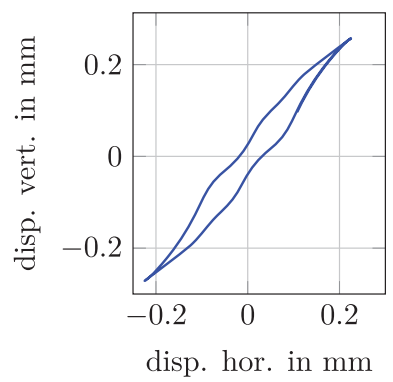

$t=0.36 \mathrm{~s}$

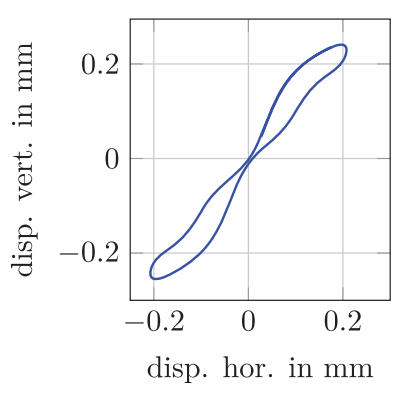

Figure 13. Transient development of the simulated orbit diagram at four points in time.

would be expected that the modes in both polarizations occur at the same eigenfrequency but, in fact, they are parted by a frequency difference $\Delta f_{g}=0.25 \mathrm{~Hz}$. This is very similar to the frequency difference between the two planes of polarization observed in the experiment (see Fig. 5). In Figure 13 the orbit diagrams of four cycles within the first second of simulation are shown. When compared to Figure 6 , the similarity of the transient variation of the orbit is evident. As the frequency difference between the two polarizations is slightly larger in the simulation, the effect of the orbit's shape variation takes place quicker.

Finally, the transient results shall be analyzed. Therefore, the transient velocity in both polarizations close to the plucked position is visualized. Figure 14 displays the transient velocity horizontally and vertically in the first $20 \mathrm{~ms}$ after the excitation. As described in Section 4, the simulation begins at the point where the string detaches from the plectrum. The simulated oscillation is very similar to the experimental results in frequency, amplitude, and shape of the oscillation in both polarizations. Consequently, the identification of the initial conditions was successful. It is noteworthy that the only parameter that is fitted in the model is the direction and magnitude of the force that causes the initial displacement. All other parameters are directly identified from the experimental results.

Furthermore, Figure 15 displays the velocity after more than half a second. The amplitude and frequency of the simulated curve are still satisfying in this plot. Hence, not only the initial condition but also the dispersion are well approximated in the model. Although the shape of the simulated curve still fits well to the experiments, the limitations of the Rayleigh damping model are visible in the transients when looking at the overtones. As stated above, the frequency difference between the two planes of polarization is slightly larger in the simulation than in the experiment and, hence, the simulated oscillation in vertical direction has a small phase shift compared to the experimental one. The highest amplitudes in the vertical velocity of experiment and simulation are split by $0.4 \mathrm{~ms}$. When taking the first measured eigenfrequency $f_{v_{\text {meas }}}=227 \mathrm{~Hz}$ this adds up to a frequency difference of $\Delta f_{v}=0.1 \mathrm{~Hz}$ between the signals. Therefore, the transients show again, that the frequency difference in the simulation is $\Delta f_{v}=0.1 \mathrm{~Hz}$ larger than in the measurement.
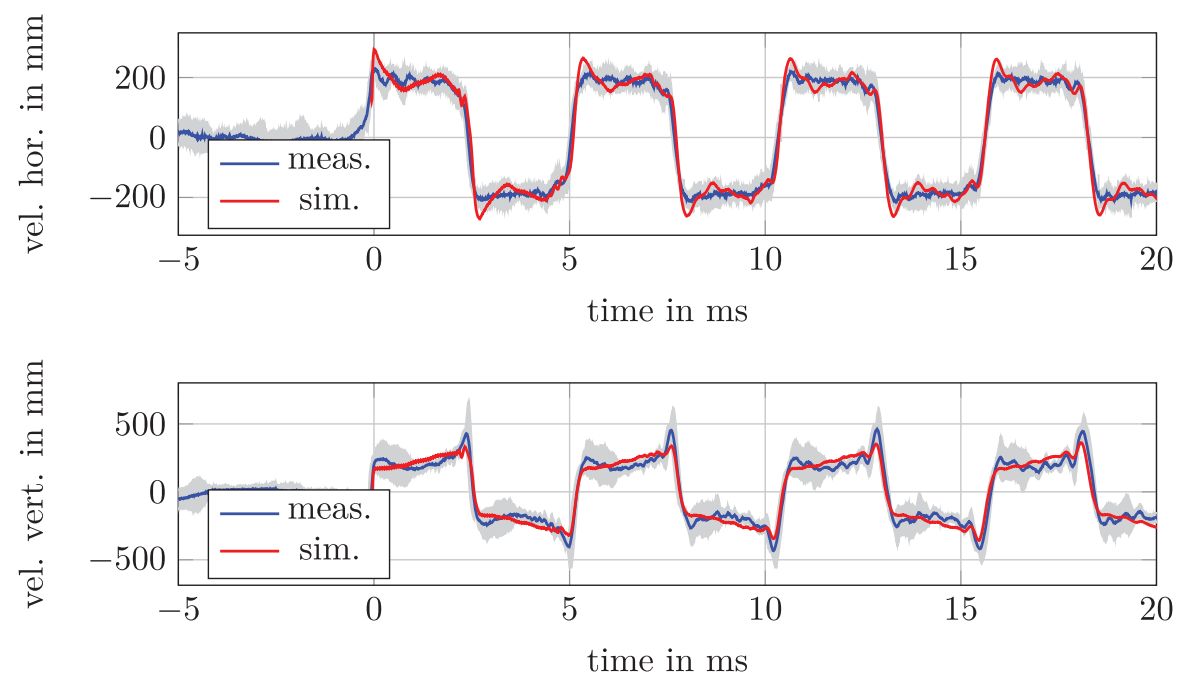

Figure 14. Velocity of experiment (blue) and simulation (red) in comparison directly after plucking in the middle. The string is measured $15 \mathrm{~mm}$ beside the middle. 

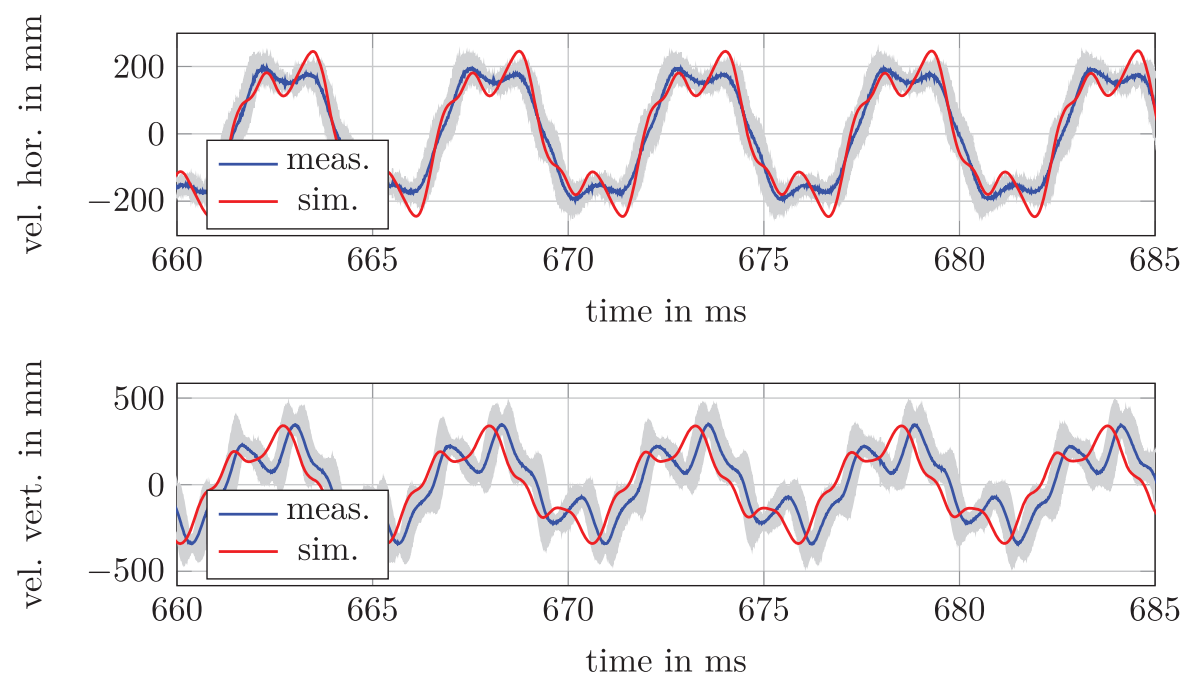

Figure 15. Velocity of experiment (blue) and simulation (red) in comparison after $660 \mathrm{~ms}$. The string is measured $15 \mathrm{~mm}$ beside the middle.

\section{Conclusion}

In this paper, the motion of a realistically plucked guitar string has been studied experimentally and numerically in both transversal polarizations. Several mechanical effects have to be taken into account. First and foremost, the string's damping is crucial for the sound of a string. Secondly, especially if the transient behavior is of interest, the stiffness of the string and the resulting dispersion has to be taken into account. Furthermore, with a realistic plucking motion it is very unlikely that only one transversal polarization of the string oscillation is excited. That leads to the need of both, an experiment with a string that can be measured in both polarizations and a numerical model that includes two transversal polarizations. Finally, the string has to be measured at different positions for the same oscillation to understand the behavior of the string as a whole.

Therefore, an experimental set-up has been developed that yields the possibility of high-fidelity measurements of the string oscillation in both polarizations. An excitation mechanism has been developed that produces a realistic and well reproducible plucking motion. This made it possible not only to measure the string's motion but also to examine the mechanically complex plucking motion in detail. This capability renders the identification of suitable initial conditions for a numerical string model possible. These initial conditions consisting of initial displacement and velocity have been identified for a realistic plucking motion. Moreover, the frequency dependent damping ratio of the string has been identified in the experiment.

The finite element software Abaqus has been used to model a string consisting of three parts. A multi-step simulation procedure is carried out to apply the initial conditions. Together with the experimental set-up this sophisticated numerical model leads to a very good approximation of the string oscillation in both transversal polarizations for a realistically plucked string. That is to say, the numerical model approximates the behavior of the string very well directly after the excitation. Even for an extended simulation time, the correlation remains very good. After more than half a second, i.e. more than 100 cycles, both shape and amplitude still correlate very well.

The model could be successfully used to vividly approximate the coupling between the oscillations in the two planes of polarization. This coupling could be traced back to a torsional movement of the string on the saddle which causes a small frequency difference between the vertical and the horizontal oscillation.

\section{Conflict of interest}

The authors state that there are no competing interests to declare.

\section{Funding}

This research did not receive any specific grant from funding agencies in the public, commercial, or not-for-profit sectors.

\section{References}

1.J. Woodhouse: On the synthesis of Guitar Plucks. Acta Acustica United With Acustica 90 (2004) 928-944.

2. P.M. Morse, K.U. Ingard: Theoretical Acoustics. Princeton University Press, Princeton, 1968.

3. S. Bilbao: Numerical Sound Synthesis: Finite Difference Schemes and Simulation in Musical Acoustics. John Wiley \& Sons, Hoboken, 2009.

4. A. Paté, J. Le Carrou, B. Fabre: Predicting the decay time of solid body electric guitar tones. The Journal of the Acoustical Society of America 135 (2014) 3045-3055. 
5. M. Ducceschi, S. Bilbao: Linear stiff string vibrations in musical acoustics: Assessment and comparison of models. The Journal of the Acoustical Society of America 140 (2016) 2445-2454.

6. A. Chaigne, A. Askenfelt: Numerical simulations of piano strings. I. A physical model for a struck string using finite difference methods. The Journal of the Acoustical Society of America 95 (1994) 1112-1118.

7. N. Giordano: Finite-difference modeling of the piano. The Journal of the Acoustical Society of America 119 (2006) 3291-3291. https://doi.org/10.1121/1.4808919.

8. J. Bensa, S. Bilbao, R. Kronland-Martinet, J.O. Smith: The simulation of piano string vibration: From physical models to finite difference schemes and digital waveguides. The Journal of the Acoustical Society of America 114 (2003) 1095-1107.

9. É. Ducasse: On waveguide modeling of stiff piano strings. The Journal of the Acoustical Society of America 118 (2005) 1776-1781.

10. J. Woodhouse: Plucked guitar transients: Comparison of measurements and synthesis. Acta Acustica United With Acustica 90 (2004) 945-965.

11. J. Chabassier, A. Chaigne, P. Joly: Modeling and simulation of a grand piano. The Journal of the Acoustical Society of America 134 (2013) 648-665.

12. C. Issanchou, S. Bilbao, J. Le Carrou, C. Touzé, O. Doaré: A modal-based approach to the nonlinear vibration of strings against a unilateral obstacle: Simulations and experiments in the pointwise case. Journal of Sound and Vibration 393 (2017) 229-251.

13. C. Issanchou, J. Le Carrou, C. Touzé, B. Fabre, O. Doaré: String/frets contacts in the electric bass sound: Simulations and experiments. Applied Acoustics 129 (2018) 217-228.

14. M. Zollner: Physik der Elektrogitarre (in German). Digitaldruckfabrik, Leipzig, 2014.

15. A. Askenfelt, E.V. Jansson: From touch to string vibrations. III: String motion and spectra. The Journal of the Acoustical Society of America 93 (1993) 2181-2196.

16. J. Pakarinen, M. Karjalainen: An apparatus for measuring string vibration using electric field sensing, in Proceedings Stockholm Music Acoustics Conference. 2003. pp. 739-742.

17. J. Le Carrou, D. Chadefaux, L. Seydoux, B. Fabre: A lowcost high-precision measurement method of string motion. Journal of Sound and Vibration 333 (2014) 3881-3888.

18. N. Plath: High-speed camera displacement measurement (HCDM) technique of string vibration, in Proceedings of the Stockholm Music Acoustics Conference. 2013. pp. 188-192.
19. J. Le Carrou, F. Gautier, N. Dauchez, J. Gilbert: Modelling of sympathetic string vibrations. Acta Acustica United With Acustica 91 (2005) 277-288.

20. A. Paté, J. Le Carrou, A. Givois, A. Roy: Influence of plectrum shape and jack velocity on the sound of the harpsichord: An experimental study. The Journal of the Acoustical Society of America 141 (2017) 1523-1534.

21. D. Chadefaux, J. Le Carrou, S. Le Conte, M. Castellengo. Analysis of the harpsichord plectrum-string interaction, in: Proceedings of the Stockholm Music Acoustics Conference (SMAC). 2013.

22. D. Chadefaux, J. Le Carrou, B. Fabre: A model of harp plucking. The Journal of the Acoustical Society of America 133 (2013) 2444-2455.

23. B. Bank, M. Karjalainen: Passive admittance matrix modeling for guitar synthesis, in Proc. Conf. on Digital Audio Effects, Graz. 2010. pp. 3-8.

24. M. Hanss, P. Bestle, P. Eberhard: A reproducible excitation mechanism for analyzing electric guitars. PAMM 15 (2015) $45-46$.

25. C.H. Hodges, J. Power, J. Woodhouse: The use of the sonogram in structural acoustics and an application to the vibrations of cylindrical shells. Journal of Sound and Vibration 101 (1985) 203-218.

26. H. Mansour, The Bowed String and its Playability: Theory, Simulation and Analysis. PhD thesis, McGill University Libraries, Montreal, QC, 2016.

27. R.D. Cook, D.S. Malkus, M.E. Plesha, R.J. Witt: Concepts and Applications of Finite Element Analysis. John Wiley \& Sons, New York, 2002.

28. C. Valette: Mechanics of Musical Instruments, Chapter The mechanics of vibrating strings. Springer, New York, 1995. pp. 115-183.

29. C. Desvages, S. Bilbao, M. Ducceschi: Improved frequencydependent damping for time domain modelling of linear string vibration, in Proceedings of Meetings on Acoustics ICA, Vol. 1, Acoustical Society of America. 2016.

30. V. Debut, J. Antunes, M. Marques, M. Carvalho: Physicsbased modeling techniques of a twelve-string Portuguese guitar: A non-linear time-domain computational approach for the multiple-strings/bridge/soundboard coupled dynamics. Applied Acoustics 108 (2016) 3-18.

31. Abaqus: Analysis User's Guide. Simulia, Providence, 2014.

32. J. Chabassier, S. Imperiale: Stability and dispersion analysis of improved time discretization for simply supported prestressed Timoshenko systems. Application to the stiff piano string. Wave Motion 50 (2013) 456-480.

Cite this article as: Brauchler A, Ziegler P \& Eberhard P. 2020. Examination of polarization coupling in a plucked musical instrument string via experiments and simulations. Acta Acustica, 4, 9. 